www.nature.com/clinicalpractice/neuro

Oswestry Disability Index questionnaire, and the VISUAL ANALOG SCALE for pain (scale 0-100).

Overall clinical success in each patient was determined by four criteria: no neurological deterioration from preoperative status, no major complications, no failure of the device, and $\geq 25 \%$ improvement in Oswestry Disability Index score at 24 months compared with the preoperative score. Overall clinical success at 24 months was significantly higher for patients who received the artificial disc than for controls $(P=0.0004)$, as was patient satisfaction $(P=0.0011)$.

The authors conclude that the CHARITÉ ${ }^{\circledR}$ artificial disc provides a safe and effective alternative to the established surgical treatment for DDD, and state that it is associated with economic benefits such as a lower rate of reoperation.

Christine Kyme

Original article Blumenthal S et al. (2005) A prospective, randomized, multicenter Food and Drug Administration investigational device exemptions study of lumbar total disc replacement with the CHARITÉ ${ }^{\mathrm{TM}}$ artificial disc versus lumbar fusion. Part I: evaluation of clinical outcomes. Spine 30: $1565-1575$

\section{Is buccal midazolam more effective than rectal diazepam for treating seizures in children?}

A study recently reported in The Lancet compared the safety and efficacy of buccal midazolam with that of rectal diazepam in children with acute seizures.

Between October 2000 and February 2004, four UK hospitals recruited children aged $\geq 6$ months who presented with an acute seizure. Patients were randomized to treatment with agedetermined doses of either buccal midazolam or rectal diazepam. The primary endpoint was therapeutic success, defined as seizure cessation within $10 \mathrm{~min}$, lasting for at least $1 \mathrm{~h}$, without respiratory depression requiring mask inflation or intubation. Lorazepam was given intravenously if seizures lasted $>10 \mathrm{~min}$; in these cases, treatment was considered to have failed.

Overall, 177 patients were treated for 219 separate seizure episodes. Buccal midazolam was more effective than rectal diazepam, even when numerous variables were adjusted for (e.g. hospital, age, prior treatments, epilepsy diagnosis and presence of fever; odds ratio 4.1, Cl 2.2-7.6;
$P<0.001$ ), and when only the initial episode for each patient was included (odds ratio $3.5, \mathrm{Cl} 1.8$ 7.0; $P=0.008$ ). Compared with those receiving rectal diazepam, fewer children receiving buccal midazolam experienced treatment failure. Buccal midazolam also ended seizures more rapidly than rectal diazepam $(P=0.01)$. The incidence of respiratory depression was not significantly different between treatment groups.

The authors conclude that buccal midazolam is more effective than rectal diazepam for the emergency treatment of children presenting with acute seizures, and that it does not increase the risk of respiratory depression.

Rebecca Ireland

Original article McIntyre J et al. (2005) Safety and efficacy of buccal midazolam versus rectal diazepam for emergency treatment of seizures in children: a randomised controlled trial. Lancet 366: 205-210

\section{Stroke severity-an important predictor of post-stroke epilepsy}

A Norwegian group has examined long-term follow-up in ischemic stroke patients to assess the prevalence of post-stroke epilepsy. The investigators also looked at the predictors for post-stroke epilepsy and the influence of treatment in stroke units on the long-term outcomes of epilepsy.

This randomized, prospective study identified 484 patients with ischemic stroke admitted to hospital between March 1994 and December 1995 within $24 \mathrm{~h}$ after stroke onset. After admission, patients were randomized to treatment in stroke units or in general medical wards. Fifty-six patients died within 1 week of admission. Data were recorded, including the results of CT and electrocardiograms and the extent of impairment and disability assessed by the Scandinavian Stroke Scale. Overall, 252 patients survived 7-8 years post-stroke and were followed up by telephone or in an outpatient setting. Medical records of those who died were also assessed.

In total, 28 patients experienced post-stroke seizures, and 15 of these developed poststroke epilepsy, defined as two or more recurrent seizures occurring $>1$ week after stroke. Severe strokes, defined as Scandinavian Stroke Scale score $<30$, were found to be a significant predictor of post-stroke epilepsy. Treatment in stroke units was not found to influence the risk of post-stroke epilepsy.

\section{GLOSSARY}

VISUAL ANALOG SCALE A line anchored by word descriptors (e.g. 'no pain' and 'very severe pain') used to assess a characteristic that ranges across a continuum of values 